\title{
Dermatomyositis following Biosimilar Trastuzumab in a Breast Cancer Patient: A Case Report
}

\author{
Minoosh Moghimia Kasra Khodadadi ${ }^{b}$ \\ aHaematology-Medical Oncology, Zanjan University of Medical Sciences, Zanjan, Iran; \\ bInternal Medicine, Zanjan University of Medical Sciences, Zanjan, Iran
}

\section{Keywords}

Anti-human epidermal growth factor receptor 2 - Breast cancer - Dermatomyositis .

Trastuzumab · Case report

\begin{abstract}
Trastuzumab, as a recombinant lgG1 kappa, is a humanized monoclonal antibody against human epidermal growth factor receptor 2. Accordingly, it is widely used in breast cancers at early and advanced stages. Dermatomyositis is a rare adverse event of trastuzumab therapy, which is not well documented yet. In this study, a patient was treated for invasive ductal carcinoma with some symptoms of rash and generalized fatigue. These symptoms started after the fifth cycle of trastuzumab, which were gradually deteriorating. This patient's medical and family histories were unremarkable. The progression of the disease was ruled out as a possible cause of dermatomyositis, and the laboratory evaluation revealed a moderate increase in serum muscle protein (CPK). So, trastuzumab treatment was discontinued, and by passing 1 month from the start of prednisolone and hydroxychloroquine, the patient had no symptoms.
\end{abstract}

\section{Introduction}

In patients with breast cancer (BC), adjuvant therapy with both trastuzumab and chemotherapy has been shown to increase both survival and response rates compared to trastuzumab alone $[1,2]$ Notably, trastuzumab is a recombinant IgG1 kappa and a humanized monoclonal antibody against human epidermal growth factor receptor 2 (HER2) [3]. Correspondingly, HER2 is usually overexpressed in $20-25 \%$ of cases with BC worldwide [4]. Trastuzumab binds to this receptor's extracellular domain and then prevents HER2 homodimerization by blocking HER2-mediated signaling. Also, this is believed to promote antibody-dependent 
Fig. 1. Reddish-purple rash on the upper eyelids (heliotrope rash).

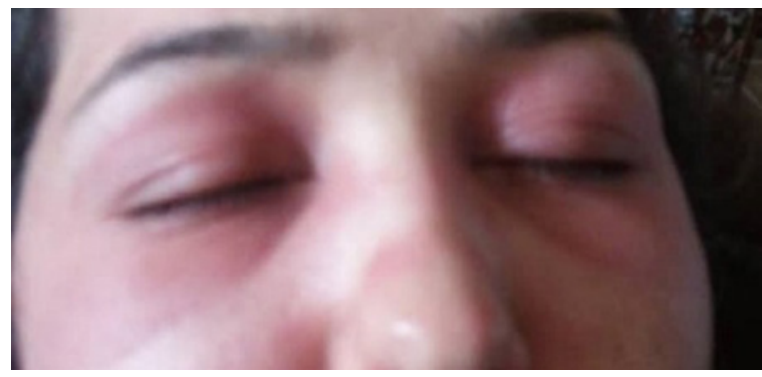

cellular cytotoxicity, resulting in HER2-expressing cells' death [5]. It is widely used in cases at early and advanced stages of BC $[6,7]$. Trastuzumab is linked to cardiotoxicity, which is usually manifested as the reduced left ventricular ejection fraction. The precise pathogenesis of this case is unclear yet, but it may include the reduced clearance of reactive oxygen species in cardiac myocytes [8]. Herein, we presented a case of a patient treated with trastuzumab, who manifested the clinical symptoms and laboratory findings of dermatomyositis (DM) with a prominent cutaneous feature.

\section{Case Report}

A 35 -year-old pregnant woman at 36 weeks of gestation with a $23 \times 11$-mm mass and multiple lymph nodes in the left axillary up to $23 \times 10$-mm involvement in ultrasonography was included in this study. After performing core needle biopsy, she had been diagnosed with invasive ductal carcinoma grade II in the upper outer quadrant of her left breast. Accordingly, this tumor was characterized as negative by immunohistochemistry for estrogen and progesterone receptors, but it resulted as positive for HER2 and Ki67 positive in $>25 \%$ of tumor cells. The patient at 38 weeks of gestation delivered, and then the assessment for metastasis was done. Fortunately, no metastases were found, and her cancer stage was found to be II.

The patient included in this study received neoadjuvant chemotherapy with both adriamycin and cyclophosphamide 4 courses. Following the breast ultrasound, clear reductions were observed in the size of the mass and axilla lymph nodes. Thereafter, the patient received paclitaxel and AryoTrust (TH) for a 12-week duration, and then AryoTrust was continued once per 3 weeks. Of note, the generic name of the trastuzumab biosimilar used for the patient was Aryo Trust. Afterward, left breast quadrectomy was done, showing no tumor residue, and left axillary sentinel lymph node excisional biopsy also resulted as negative (which is a very good response to neoadjuvant chemotherapy). After performing breast-conserving surgery, radiation therapy to the whole breast and regional lymphatics were considered. This technique contains tangential fields that is performed using the 3-dimensional conformal radiation therapy. The dose provided to the whole breast was between 45 and 50 Gy over 5-6 weeks (1.8-2 Gy tumor dose daily, 5 weekly fractions), with an additional boost dose or 10-16 GY in 2 Gy fractions to the tumor bed with the patient treated with 6-MV photons.

The overall treatment was well tolerated by the patient. After performing 5 cycles of AryoTrust ( $6 \mathrm{mg} / \mathrm{kg}$ every 3 weeks), she complained of swollen eyes and urticarial lesions. By conducting the physical examination, periorbital swelling and erythema (heliotrope rash) were revealed (Fig. 1). An erythematous rash on the arms (Fig. 2) and chest was also observed. Also, muscle strength of the lower limbs decreased (4/5), while it was normal in all upper limbs. The laboratory examination revealed moderately elevated serum muscle enzymes (a creatine phosphokinase value: 492 IU/L with a normal laboratory range of 38-176 IU/L;lactatedehydrogenase value: $510 \mathrm{IU} / \mathrm{L}$ with a normal laboratory range of 140-280 IU/L),

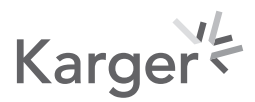


Fig. 2. An erythematous rash on the left arm.

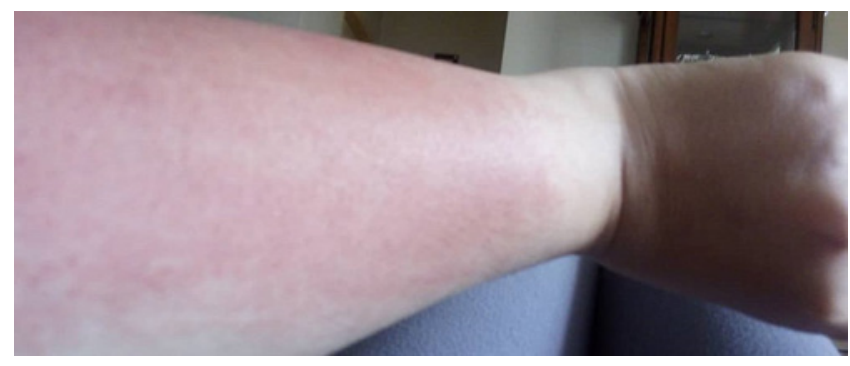

but fluorescent antinuclear antibody, anti-double-stranded DNA, and antibodies to histidyl tRNA synthetase were negative. Skin lesion biopsy diagnosed papillary edema, epidermal spongiosis with few eosinophilic infiltrations, and a mild interstitial mucin deposition. Since the interval between radiotherapy and the onset of the symptoms was about 6 months and a recent screening for disease recurrence resulted as negative, the observed DM symptoms were attributed to trastuzumab. For this reason, the practitioner discontinued the use of trastuzumab. With Rheumatologic consult, prednisolone and hydroxychloroquine were started. Subsequently, the patient's symptoms improved, and 1 month later, she was free of any related symptom, and CPK was found in the normal range. In the first week, AryoTrust was started with $4 \mathrm{mg} / \mathrm{kg}$, which was then followed by $2 \mathrm{mg} / \mathrm{kg}$ weekly for 3 weeks. Afterward, the dose of AryoTrust was increased to $6 \mathrm{mg} / \mathrm{kg}$ every 3 weeks. Fortunately, the patient had no complications.

\section{Discussion}

This was the first report of biosimilar trastuzumab-induced DM. Correspondingly, the other case reports were related to a brand form of trastuzumab (Herceptin). Trastuzumab was shown to have a significant effect on the treatment of HER2 overexpressing (HER2positive) BC $[9,10]$. Notably, cutaneous adverse reactions to trastuzumab are rare. In a study by Seidler et al. [10] who analyzed drug-induced DM cases, the results showed that $44 \%$ of subjects had an underlying malignancy, which usually occurs within the first 5 years after diagnosis. Also, the onset of drug-induced DM symptoms on average starts within 24 months of treatment. The study also showed that several people who developed drug-induced DM had no improvement with drug discontinuation, and they needed a special treatment for DM [10]. Skin adverse reactions in those patients receiving trastuzumab have been reviewed in various articles, as follows:

1. Radiation therapy-associated acute skin toxicity (dermatitis) as well as late skin reactions (telangiectasia, local pain, and fibrosis) [11, 12].

2. Flagellate erythema [13].

3. Infusion reaction (this occurs in $30-40 \%$ of patients, usually presented with chills or fever, with the first infusion, which occur only in 3-5\% of patients with subsequent infusions. Severe infusion reactions are uncommon [about $0.3 \%$ ], which may include rash) [14-17].

4. Nail toxicity (including softening, thinning or loss, paronychia, and discoloration [18]).

5. Photosensitivity $[19,20]$.

6. Skin toxicity, including eruptions on the face and body, skin detachment or thinning on hands and feet itching, and skin drying [18].

7. Tufted hair folliculitis [21].

8. Dermatomyositis [22].

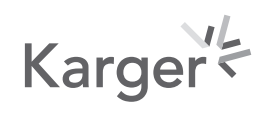


In the current study, because the patient's symptoms have started by passing 6 months from the radiotherapy and the possibility of recurrence was ruled out, the symptoms were attributed to AryoTrust. According to the consultation with a Rheumatologist, the treatment with both prednisolone and hydroxychloroquine was started. In this regard, the dose was gradually increased, and no similar symptoms were seen.

\section{Conclusion}

DM can be considered as a complication of biosimilar trastuzumab. The best approach is to discontinue the drug. If the symptoms of DM continue, medication can be started, and after the symptom improvement with a careful follow-up, the drug can be re-started, and the patient can be followed up with a gradual increase of biosimilar trastuzumab.

\section{Statement of Ethics}

This study was approved by the institutional ethics review boards of our university (Approval No. IR.ZUMS.REC.1400.057). Written informed consent was obtained from the patient for publication of this case report and any accompanying images.

\section{Conflict of Interest Statement}

There are no conflicts of interest.

\section{Funding Sources}

This study was not funded.

\section{Author Contributions}

Moghimi and Khodadadi have equally contributed in designing, reviewing, drafting, analyzing the data, and writing the manuscript.

\section{Availability of Data and Material}

The authors declare that data supporting the findings of this study are available within the article.

\section{References}

1 Boekhout AH, Beijnen JH, Schellens JHM. Trastuzumab. The oncologist; 2011. p. 800-10.

2 Menard S, Pupa SM, Campiglio M, Tagliabue E. Biologic and therapeutic role of HER2 in cancer. Oncogene. 2003;22(42):6570-8.

3 Wong SF. Cetuximab: an epidermal growth factor receptor monoclonal antibody for the treatment of colorectal cancer. Clin Ther. 2005;27(6):684-94. 
4 Arteaga CL, Sliwkowski MX, Osborne CK, Perez EA, Puglisi F, Gianni L. Treatment of HER2-positive breast cancer: current status and future perspectives. Nat Rev Clin Oncol. 2011;9(1):16-32.

5 Namboodiri AM, Pandey JP. Differential inhibition of trastuzumab- and cetuximab-induced cytotoxicity of cancer cells by immunoglobulin G1 expressing different GM allotypes. Clin Exp Immunol. 2011;166(3):361-5.

6 Cardoso F, Costa A, Norton L, Senkus E, Aapro M, André F, et al. ESO-ESMO 2nd international consensus guidelines for advanced breast cancer (ABC2). Breast. 2014;25(10):1871-88.

7 Senkus, E, Kyriakides S, Ohno S, Penault-Llorca F, Poortmans P, Rutgers E, et al. Primary breast cancer: ESMO clinical practice guidelines for diagnosis, treatment and follow-up. Ann Oncol. 2015;26(Suppl 5):v8-30.

8 Mohan, N, Shen Y, Endo Y, ElZarrad MK, Wu WJ. Trastuzumab, but not pertuzumab, dysregulates HER2 signaling to mediate inhibition of autophagy and increase in reactive oxygen species production in human cardiomyocytes. Mol Cancer Ther. 2016;15(6):1321-31.

9 Pérez-Soler RTJ. HER1/EGFR targeting: refining the strategy. Oncologist. 2004;9(1):58-67.

10 Seidler AM, Gottlieb AB. Dermatomyositis induced by drug therapy: a review of case reports. J Am Acad Dermatol. 2008;59(5):872-80.

11 Caussa L, Kirova YM, Gault N, Pierga JY, Savignoni A, Campana F, et al. The acute skin and heart toxicity of a concurrent association of trastuzumab and locoregional breast radiotherapy including internal mammary chain: a single-institution study. Eur J Cancer. 2011;47(1):65-73.

12 Meattini I, Cecchini S, Muntoni C, Scotti V, De Luca Cardillo C, Mangoni M, et al. Cutaneous and cardiac toxicity of concurrent trastuzumab and adjuvant breast radiotherapy: a single institution series. Med Oncol. 2014; 31(4):891.

13 Cohen PR. Trastuzumab-associated flagellate erythema: report in a woman with metastatic breast cancer and review of antineoplastic therapy-induced flagellate dermatoses. Dermatol Ther. 2015;5(4):253-64.

14 Baselga JJ. Clinical trials of Herceptin $\circledast$ (trastuzumab). Eur J Cancer. 2001;37:18-24.

15 Smith IE. Efficacy and safety of Herceptin in women with metastatic breast cancer: results from pivotal clinical studies. Anticancer Drugs. 2001;12:S3-10.

16 Laux I, Jain A, Singh S, Agus DB. Epidermal growth factor receptor dimerization status determines skin toxicity to HER-kinase targeted therapies. Br J Cancer. 2006;94(1):85-92.

17 Myskowski PL, Halpern AC. Skin reactions to the new biologic anticancer drugs. Curr Opin Support Palliat Care. 2009;3(4):294-9.

18 Adachi S, Yoshimura T, Matsuoka T, Okada K, Yasuda T, Kamei K. Appearance of skin and nail toxicity in patients with breast cancer who underwent trastuzumab-containing chemotherapy. Gan To Kagaku Ryoho. 2011;38(9):1453.

19 Cohen AD, Mermershtain W, Geffen DB, Schoenfeld N, Mamet R, Cagnano E, et al. Cutaneous photosensitivity induced by paclitaxel and trastuzumab therapy associated with aberrations in the biosynthesis of porphyrins. J Dermatol Treat. 2005;16(1):19-21.

20 Akay BN, Unlu E, Buyukcelik A, Akyol A. Photosensitive rash in association with porphyrin biosynthesis possibly induced by docetaxel and trastuzumab therapy in a patient with metastatic breast carcinoma. Jpn J Clin Oncol. 2010;40(10):989-91.

21 Rosman IS, Anadkat MJ. Tufted hair folliculitis in a woman treated with trastuzumab. Target Oncol. 2010;5(4): 295-6.

22 Trontzas IP, Syrigos NK, Kotteas EA. A case of trastuzumab-induced dermatomyositis; 2020. 